of allatotropin should be a better source. Small amounts of released hormone for confirmatory studies can probably be obtained from these glands by stimulation of the nerve leading to them or by high $\mathrm{K}^{+}$ depolarization as has been done with the diuretic hormone of Rhodnius (Maddrell \&
Gee, J. Exp. Biol. 61, 115; 1974). Also the identification of the PTTH-secreting cell makes possible a detailed neurophysiological study of the control of the release of this hormone by various internal and external signals such as larval size, photoperiod, and temperature.

\title{
Dielectric relaxation spectroscopy
}

\section{from J. H. Calderwood}

If the permittivity (or refractive index) of a dielectric material is measured over a frequency range extending from very low values up to the infrared, a number of abosorptions will be observed. Frequencies at which these absorptions occur, and their strengths, may be a decisive factor in determining the practical use to which the materials can be put. Study of the spectra can yield information about the structure of the molecules of the material, and of the molecular dynamics of the system which the molecules, and in some case aggregates of molecules, comprise. These studies and their applications in science and industry were the theme of a recent conference.*

The interpretation of the dielectric measurements presents further problems. H.A. Price of the University College of Wales, Aberystwyth, pointed out that although isotropic liquids usually show only one absorption maximum in their relaxation spectrum, it may be broader than the absorption which would be predicted by the Debye equation. In some cases, the broadening is sufficient to allow the relaxation to be resolved into multiple processes corresponding to whole molecule rotation and the internal rotation of a flexible group within the molecule. Debye considered spherical molecules, but if his treatment is generalised to ellipsoidal molecules as was done by Perrin, then separate relaxation processes are predicted corresponding to rotation about the different molecular axes. Price gave details of the observation of multiple relaxation processes for some liquid crystals, and the particular cases of p-methoxy-benzylidene -p-n-butylaniline (MBBA) and n-heptylcyanobiphenyl (7CB).

R. Toomer from the University College of North Wales, Bangor, was concerned with the separation of conduction and relaxation processes as energy loss in a dielectric can be caused by either of them. Metal electrodes are normally used for dielectric measurements, but as they are capable of injecting charge into dielectric solids, thus initiating a conduction process, it is necessary to separate that conduction from the dielectric relaxation phenomena. Toomer described an experimental technique whereby a material could be polarized by

*The conference on 'Dielectric relaxation spectroscopy in Science and Engineering' was held at the Paul Langevin Centre
Aussois, France in March, 1980 . induction, without any direct metallic contact, and the surface scanned by a sensitive induction probe, again without contact being made, to determine the time decay of the surface potential, and so determine the relaxation time of the specimen.

Another technique, especially suitable for the investigation of charge storage mechanisms, is that of field-induced thermally stimulated currents (FITSC). It involves raising of the temperature of a specimen at a known rate while the current is measured. Vanderschueren of the University of Liège gave a critical account of how such measurements should be interpreted and paid particular attention to the tests that should be carried out to ensure that spurious effects are absent. In particular, a systematic variation of experimental parameters which might affect the results should be carried out: these include such factors as electrode material, specimen thickness and field intensity. Other techniques involving thermal relaxation should also be used to confirm the results of FITSC.

The more traditional method of the examination of the frequency spectrum can be used to investigate the behaviour of aggregates of melecules of the micelle type. R. Pottel of the University of Göttingen described measurements in the frequency range $0.1 \mathrm{MHz}$ to $60 \mathrm{GHz}$ on liquid crystalline aqueous systems and showed how the dynamical behaviour could be influenced by the molecules of the solvent, and by the solute concentration. Molecular dynamics can also be studied by the rather unusual technique of non-linear dielectric spectroscopy, when materials are polarized by fields of high strength. If the polarization is expressed by a power series, the second and higher order of coefficients characterise the non-linear properties. Helleman of the University of Leuven described how the non-linear effects observed for reactive systems are an indication of the extent to which the field has perturbed the chemical composition of the sample. Thus from the relaxation of the non-linear effect, information on chemical rates can be collected. This is a very unusual tool to be employed for the investigation of chemical rate processes.

A portion of the frequency spectrum which is more difficult than most is the millimetre wave region. At longer wavelengths, waveguides, lines, closed cavities, and bridges are available, while at shorter wavelengths optical techniques become applicable. A. Gebbie and P. R. Mason of Imperial College, London, described how over-moded cavities could be used in the millimetre region. The overmoding is produced by means of a rotating paddle in the cavity which scatters the radiation in all directions, thus ensuring that the specimen will be subjected to its influence, without the possibility of its being affected by a special wave pattern. The open resonator provides another very useful means of measurement in the microwave region, and its advantages become particularly significant as the wavelength decreases. A. L. Cullen of University College, London, described the construction of an open resonator suitable for measuring the permittivity of a material in the form of a sheet. In order to assess the error which might result from some small departure from the ideal in a design feature, the interesting technique was adopted of deliberately making the departure large, and making measurements as it was progressively reduced. A. C. Lynch, also of University College, discussed the limitation in the accuracy of permittivity measurements and showed how some of the difficulties might be overcome. For example, in a microwave resonator it is possible to arrange that the surface of the sheet specimen are in a field which is nearly zero, thus reducing the effect of any error in making the most difficult measurement associated with the experiment. Surprisingly, that is the measurement of the thickness of the specimen!

Even when we know how to obtain good results, and how to interpret them, the problem remains of how to predict them. Prediction is made possible by the construction of models which corresponded in some analogous way with the physical reality. Although in principle the construction of such models might be fairly straight forward the analysis of the model to determine the behaviour which it predicts is usually a matter of considerable technical complexity. The problems associated with modelling dielectric behaviour were discussed by M. W. Evans of the University College of Wales, Aberystwyth, P. Bordewijk of Leiden University, and C. Brot of the University of Nice. Computer simulation is liable to be very expensive in computer time, but Brot showed how reliable values of complex permittivity of highly polar fluids could be obtained by a two-dimensional simulation involving only a few hundred molecules, despite the long-range character of dipolar interaction. This is an encouraging advance in a field in which progress is not easy.

J. H. Calderwood is Professor of Engineering at the University of Salford. 